\title{
LUPUS SCIENCE\& MEDICINE \\ Prediction of prognosis and renal outcome in lupus nephritis
}

\author{
loannis Parodis $\left(10,{ }^{1,2}\right.$ Farah Tamirou, ${ }^{3,4}$ Frédéric A Houssiau ${ }^{3,4}$
}

To cite: Parodis I, Tamirou F, Houssiau FA. Prediction of prognosis and renal outcome in lupus nephritis. Lupus Science \& Medicine 2020;7:e000389. doi:10.1136/ lupus-2020-000389

Received 4 February 2020 Accepted 6 February 2020

\section{Check for updates}

(c) Author(s) (or their employer(s)) 2020. Re-use permitted under CC BY-NC. No commercial re-use. See rights and permissions. Published by BMJ.

${ }^{1}$ Division of Rheumatology, Department of Medicine Solna, Karolinska Institutet, Stockholm, Sweden

${ }^{2}$ Rheumatology, Karolinska University Hospital, Stockholm, Sweden

${ }^{3}$ Pôle de Pathologies

Rhumatismales Inflammatoires et Systémiques, Institut de Recherche Expérimentale et Clinique, Université catholique de Louvain, Brussels, Belgium ${ }^{4}$ Rheumatology Department, Cliniques universitaires SaintLuc, Brussels, Belgium

Correspondence to

Professor Frédéric A Houssiau; frederic.houssiau@uclouvain.be

\section{ABSTRACT}

Lupus nephritis (LN) is a severe manifestation of SLE, characterised by subendothelial and/or subepithelial immune complex depositions in the afflicted kidney, resulting in extensive injury and nephron loss during the acute phase and eventually chronic irreversible damage and renal function impairment if not treated effectively. The therapeutic management of $L N$ has improved during the last decades, but the imperative need for consensual outcome measures remains. In order to design trials with success potentiality, it is important to define clinically important short-term and longterm targets of therapeutic and non-therapeutic intervention. While it is known that early response to treatment is coupled with favourable renal outcomes, early predictors of renal function impairment are lacking. The information gleaned from kidney biopsies may provide important insights in this direction. Alas, baseline clinical and histopathological information has not been shown to be informative. By contrast, accumulating evidence of pronounced discrepancies between clinical and histopathological outcomes after the initial phase of immunosuppression has prompted investigations of the potential usefulness of per-protocol repeat kidney biopsies as an integral part of treatment evaluation, including patients showing adequate clinical response. This approach appears to have merit. Hopefully, clinical, molecular or genetic markers that reliably reflect kidney histopathology and portend the long-term prognosis will be identified. Novel non-invasive imaging methods and employment of the evolving artificial intelligence in pattern recognition may also be helpful towards these goals. The molecular and cellular characterisation of SLE and LN will hopefully result in novel therapeutic modalities, maybe new taxonomy perspectives, and ultimately personalised management.

\section{INTRODUCTION}

Lupus nephritis (LN) is one of the most severe complications in SLE, affecting 35\%-60\% of the patients depending on ethnicity, sex and age of disease onset among other factors. ${ }^{1-4}$ Genetic and nephron cargo, SLE disease course and kidney toxicity, for example, drug-induced toxicity, contribute to long-term impairment of the renal function. Importantly, LN flares constitute a major cause of nephron loss, cumulatively contributing to renal function deterioration.

Current therapies for $\mathrm{LN}$ are not sufficiently efficacious in inducing remission or preventing new flares and not all patients show adequate treatment responses. In fact, fewer than $30 \%$ achieve complete remission within 6 months of therapy. ${ }^{56}$ Despite decelerating rates over the last decades, up to $20 \%$ of patients who have been afflicted by LN will ultimately develop end-stage kidney disease within the first decade of their disease course. ${ }^{7-11}$ For this reason, prediction of the long-term renal outcome at early stages of the disease is of vital importance. Thus, several studies have sought to identify early clinical features, laboratory tests and molecular mechanisms that are associated with unfavourable renal prognosis, in order to optimise the surveillance and interventions in these patients.

The targets of management of patients with LN can be divided into short-term (prevention of flares) and long-term targets (prevention of renal function impairment). Current research in the field focuses on identification of clinical, histological and molecular markers of activity, damage, treatment response and renal prognosis.

\section{CLINICAL PREDICTORS}

Baseline clinical and laboratory clinical data do not predict renal outcome

Different investigations have examined clinical and serological markers as predictors of renal prognosis. The information retrieved at the time of active renal disease (baseline), including proteinuria and conventional serological markers, has not shown ability to adequately predict the long-term kidney outcome, in terms of renal function. This was for example the case in two randomised clinical trials, the MAINTAIN Nephritis Trial ${ }^{12}$ and the Euro-Lupus Nephritis Trial, ${ }^{13}$ as shown in post hoc analyses by Tamirou et $a l^{14}$ and Dall'Era et $a l,{ }^{15}$ respectively.

\section{Early response predicts good long-term renal prognosis}

By contrast, early decrease of proteinuria levels over 6 months of treatment has shown ability to predict a more favourable long-term renal outcome compared with patients with 
persistently high-grade proteinuria, ${ }^{16}$ and a recent series of three studies of similar design concluded that the most useful clinical predictor of good long-term renal outcome (defined using a cut-off for serum concentrations of creatinine at 7 years after the LN episode) is proteinuria levels $<0.7-0.8 \mathrm{~g} /$ day at month 12 from treatment initiation. ${ }^{141517}$ Nevertheless, while this clinical target could identify patients who had a good renal outcome with a high positive predictive value, it could not predict the outcome of patients who did not achieve the target since the negative predictive value of this proteinuria cut-off was low in two of the three investigations. ${ }^{1415}$ Thus, the challenge to find appropriate predictors remains, at least predictors of unfavourable renal prognosis.

\section{Persistent microscopic haematuria does not add prognostic value}

Cellular casts in the microscopic analysis of the urinary sediment are included in SLE classification criteria sets $^{1819}$ and constitute an important diagnostic tool to raise suspicion of kidney involvement. Importantly, findings in the urinary sediment differ substantially between proliferative and non-proliferative glomerulonephritis, with haematuria being mostly associated with proliferative disease,${ }^{20}$ pointing to another diagnostic utility of the urinalysis.

Urinalysis may also be useful in differential diagnostics between disease entities, based on, for example, whether proteinuria or haematuria is the predominant aberrance. For example, while proteinuria typically is the main finding in urinalysis in LN, haematuria is a more prominent abnormality in antineutrophil cytoplasmic antibody-associated vasculitis. In the latter, haematuria mainly derives from glomerular endothelial cell and surface layer injuries, resulting in necrotising and crescentic glomerulonephritis and ultimately a severe impairment of the glomerular filtration barrier. ${ }^{21}$

However, the usefulness of the urinalysis in clinical studies has in recent years been debated; the main reason for that being the fact that quantification of its components is characterised by a profound variability between assessors and between consecutive measurements. Moreover, urinalysis has been withdrawn in the newly proposed 2019 European League Against Rheumatism/American College of Rheumatology classification criteria for SLE. ${ }^{22}$

More importantly, results from urinalysis, in particular haematuria either before the initial immunosuppressive therapy or at 1 year from treatment initiation, have not shown prognostic ability regarding the long-term kidney outcome, and addition of such results to the proteinuria response did not improve the prognostic value of proteinuria $^{15}$ or even worsened it. ${ }^{14}$ Additionally, haematuria has in previous investigations shown weak or no correlation with activity features in kidney biopsies. ${ }^{23}$

One of the reasons for this is undoubtedly the fact that different laboratories analyse the urine sediment differently, and thus, no homogenised data can be retrieved for use in trials. Moreover, the urine volume may affect the accuracy of the measurements of cellular counts, with large volumes resulting in greater dilutions of the cellular elements in the pellets. In the same manner, the time of the collection may affect the dilution grade for elements of importance, normally with higher concentrations in the first morning void compared with random collections during the rest of the day. While the aforementioned obstacles could be overcome with clear instructions and standardisation, other important barriers include the multiple reasons for haematuria, especially in female patients, and the high grade of subjectivity during the assessment. Indeed, not all red cells are indicative of glomerular injury, and accurate recognition of dysmorphic acanthocytes of glomerular origin contra red blood cells originating from the lower urinary tract needs special training.

Using more sophisticated approaches such as multivariable Cox proportional hazards regression models, Mackay et $a l^{24}$ recently developed panels of routine clinical, laboratory and histological parameters (termed hazard index tools) to predict key long-term outcomes, that is, new or progressive chronic kidney disease, severe kidney injury and permanent kidney replacement. It is worth noting that proteinuria and serum creatinine at month 12 were found to be important components in these tools, whereas urinalysis was not shown to add value.

\section{HISTOLOGICAL PREDICTORS Diagnostic kidney biopsy}

At clinical suspicion of $\mathrm{LN}$, it is important to perform a kidney biopsy in order to determine the LN class and exclude mimicking conditions, such as antiphospholipid antibody or antiphospholipid syndrome-associated nephropathy, IgA nephropathy, hypertensive nephrosclerosis, diabetic nephropathy and thin basement membrane disease. This information dictates the decision of treatment. ${ }^{25-27}$ Indeed, proliferative (2003 International Society of Nephrology/Renal Pathology Society (ISN/RPS) class III and IV) and membranous (2003 ISN/ RPS class V) LN differ with regard to the long-term renal outcome, thus demanding different surveillance and therapeutic management. ${ }^{28}$ By contrast, a previous study of 98 patients with incident proliferative LN showed no difference across subclasses within the proliferative spectrum (2003 ISN/RPS class III, IV-S and IV-G) regarding long-term prognosis. ${ }^{10}$ In fact, the $\mathrm{S}$ (for segmental) and $\mathrm{G}$ (for global) designations have been suppressed in the new proposed revision of the ISN/RPS classification, ${ }^{29}$ currently awaiting embracement.

The importance of the National Institutes of Health (NIH) chronicity index in the initial kidney biopsy in portending long-term prognosis was demonstrated by Austin et al as early as in $1983,{ }^{30}$ which however was not confirmed later by the Lupus Nephritis Collaborative Study Group ${ }^{31}$ or in more recent retrospective data from the LN database of the Université catholique de Louvain 
(unpublished). This point is further discussed in the repeat biopsy section.

The initial kidney biopsy also provides information about the afflicted domains within the kidney, that is, the extent of the injury in the glomerular versus the tubulointerstitial compartment. Although current classification sets mainly focus on glomerular lesions, the importance of tubulointerstitial injury and damage in short-term and long-term prognosis has been repeatedly highlighted in the literature. ${ }^{32-37}$ Proteinuria, immune complex deposition in the interstitium, proinflammatory molecules on renal tubular cells and rupture of the Bowman's capsule and cryptic antigen presentation by juxtaglomerular cells are some of the insults resulting in interstitial infiltration by inflammatory cells and, ultimately, tubular atrophy, ${ }^{38-43}$ collectively constituting a strong rationale for inclusion of the tubulointerstitial compartment in classification sets, prognostic markers and outcome measures.

\section{The role of the repeat kidney biopsy}

The role of the repeat kidney biopsy in patients with LN has been discussed rigorously during the last decades, but consensus among researchers and physicians has yet to be established. Before elaborating on the role of the repeat biopsy, it is important to make clear distinctions between different scenarios in which such repeat biopsies can be performed and how nomenclature has been used in the literature. As discussed in a recent editorial by Anders, ${ }^{44}$ five different scenarios could be described by the term 'repeat biopsy', that is, the per-protocol repeat biopsy at a predefined time point for treatment evaluation and new decision of therapy, the partial response repeat biopsy for distinguishing between residual activity and delayed healing and guide treatment accordingly, the flare repeat biopsy, the repeat biopsy to support withdrawal of the immunosuppressive treatment and the chronic kidney disease (CKD) progression repeat biopsy to determine the grade of nephrosclerosis contra treatable active injury.

Even if the nomenclature and definitions have not been used uniformly in studies of repeat kidney biopsies, several investigations have shown a discordance between clinical and histological outcome after the initial phase of immunosuppressive therapy for LN. More specifically, most studies reporting results from repeat biopsies have shown that residual renal activity may be evident in repeat biopsies from a considerable proportion of patients who have shown complete clinical responses to treatment, the latter mainly based on the proteinuric outcome. ${ }^{45-49}$ Again, as discussed above, haematuria levels have been demonstrated to yield weak or no correlations with activity components at the level of tissue in both initial and control kidney biopsies. ${ }^{23}$

The discrepant patterns between clinical and histological data at the time of the repeat kidney biopsy have prompted investigations on the role of the tissue-level information in tailoring treatment and portending the long-term kidney outcome. While the former question has yet to be addressed in prospective studies, several studies have attempted to address the latter one.

Associations between chronic tissue damage in repeat kidney biopsies and long-term impairment of the renal function have been demonstrated in both European and Hispanic LN populations. ${ }^{45} 47$ Nevertheless, this was not confirmed in another study,${ }^{50}$ indicating a need for validation. The role of residual activity in repeat kidney biopsies as a marker of the long-term kidney outcome is even less clear. Thus, the idea of a prospective multicentric study of per-protocol repeat kidney biopsies to provide evidence for optimised surveillance and management receives indeed increasing embracement within the LN researcher community. ${ }^{44}$ In this direction, a recent retrospective investigation of incident cases of proliferative LN demonstrated that different histological components in per-protocol repeat kidney biopsies showed ability to portend renal relapses and long-term renal function impairment (unpublished data). In this study, high NIH activity index scores in the repeat kidney biopsies were predictive of subsequent relapses, especially activity in the glomerular compartment, and high NIH chronicity index scores were associated with poor long-term renal prognosis, especially chronic damage in the tubulointerstitial compartment. The discrepancies with regard to the association between chronic damage in the initial kidney biopsy and long-term impairment of the renal function across studies may be due to differences in study design, as well as, importantly, due to improvements in the diagnosis and management of LN in the last decades, resulting in less kidney damage accrued in recent compared with earlier studies. Thus, the chronic changes in repeat kidney biopsies of recent studies, especially in patients who failed to respond to immunosuppressive therapy, may be similar in amount and prognostic attributes to the respective changes in initial biopsies of earlier investigations.

Altogether, accumulating evidence strongly supports the usefulness of repeat kidney biopsies as an integral part of treatment evaluation, including LN patients showing adequate clinical response. Thus, a new prospective study is currently being designed within the frame of the Lupus Nephritis Trials Network and will be entitled 'Per-protocol repeat kidney biopsy in incident cases of lupus nephritis' or, shortly, REBIOLUP. The objectives of the project will be to determine the percentage of LN patients in pathological remission after 12 months of standard of care immunosuppression, correlate histological and immunological (based on immune deposits) response to therapy with clinical response and evaluate whether therapeutic decisions steered by the results of a per-protocol repeat kidney biopsy improve renal outcomes compared with a matched control group of patients who will not undergo repeat kidney biopsy.

In brief, patients with an incident biopsy-proven proliferative or membranous LN, or combinations thereof, selected to be initiated at standard of care immunosuppressive therapy with either mycophenolate mofetil or intravenous cyclophosphamide according to the 
Euro-Lupus regimen ${ }^{13}$ (combined with glucocorticoids and ACE inhibitors or angiotensin II receptor blockers in both cases) will be eligible to be enrolled in the study. At baseline, patients will be randomised 1:1 to either undergo or not undergo a per-protocol repeat kidney biopsy at month 12 from baseline. In patients with 2003 ISN/RPS class III/IV $( \pm V)$ at baseline and an NIH activity index score $>3$ (cut-off based on retrospective unpublished data) in the repeat kidney biopsy, the immunosuppressive therapy will be intensified based on the physician's and patient's shared decision. In patients with pure membranous (2003 ISN/RPS class V) LN at baseline, individual assessment of the repeat biopsy will steer the decision of treatment, based on, for example, evaluation of immune deposits in electron microscopy or spike formation. Results from this study, including centralised evaluation of electron microscopy in baseline and repeat kidney biopsies, are anticipated to generate data on how to evaluate response to therapy in pure membranous $\mathrm{LN}$, as well as the value of the information retrieved from repeat kidney biopsies in portending long-term renal prognosis. Patients who have not undergone a repeat biopsy will be treated according to standard clinical parameters and, finally, percentages of complete renal response at month 24 and renal impairment at month 60 will be compared between the two study arms.

\section{MOLECULAR BIOMARKERS AND TISSUE-BASED APPROACHES}

In parallel with drug research, identification of serum and urinary non-invasive biomarkers that accurately reflect renal activity at the level of tissue and predict long-term renal outcome have been a topic of extensive study during the last decades. ${ }^{51}$ Investigations include single-molecule evaluations ${ }^{5-57}$ and large semi-unbiased proteomic screenings ${ }^{58}$ of serum markers, while urinary biomarkers receive increasing interest ${ }^{59-62}$ for two important reasons. First, molecules in urine are likely to be directly excreted from the kidney, which makes them highly relevant in the context of LN and other renal disorders. Second, urine samples are readily accessible for examination, and standardisation and commercialisation of urinary markers as diagnostic and/or predictive tools would therefore mean a paramount advance in the management of LN. Recent auspicious implications from single-cell RNA-sequencing pointed to strong correlations between gene expression of immune cells in urine specimens and corresponding kidney leukocytes, ${ }^{63}$ indicating that urinary biomarkers may prove useful as a surrogate for kidney biopsies in the near future.

Identification of clinical markers that portend the longterm prognosis of the lupus kidney is anticipated to lead to treat-to-target approaches in the management of $\mathrm{LN}$ in the near future. However, the knowledge gap regarding LN pathogenesis and molecular pathways underlying clinical phenotypes remains a concern. Several recent efforts have therefore focused on deepening our understanding of the molecular events that occur in patients with LN during active and quiescent phases, as well as differences with other renal disorders and non-renal SLE. In a recent investigation, Pamfil et $a l^{64}$ studied intrarenal molecular profiles associated with disease severity using high-throughput transcriptomic approaches on kidney biopsies from two independent cohorts of patients with $\mathrm{LN}$ and cadaveric donors. Adding important information to current knowledge, the researchers of this study found decreased kidney function in patients with histological evidence of tubular damage and transcriptomic patterns consistent with $\mathrm{T}$ and $\mathrm{B}$ cell activation in the kidney, which was corroborated using immunohistochemistry. By contrast, no association between decreased kidney function and systemic disease activity or activity and chronicity in the glomerular compartment was confirmed. In another study of intrarenal transcript expression by Parikh et $a l,{ }^{65}$ repeat kidney biopsies were used to associate differentially expressed genes in patients who showed good versus poor clinical response. The researchers found that transcripts associated with $\mathrm{T}$ cell activation, complement signalling pathways and type I and II interferon signature discriminated responders from non-responders and took advantage of the serial biopsies to generate a gene signature that discriminated responders from non-responders based on changes in transcript expression following treatment.

Using a more sophisticated approach, another recent study by Panousis et $a l^{66}$ combined genetic and transcriptome analysis in whole blood samples from patients with SLE and age-matched and sex-matched healthy controls. In a subanalysis, the researchers compared patients with active renal versus active non-renal SLE and found differentially expressed genes enriched in granulocyte activation and antimicrobial humoural response, whereas combining differentially expressed genes in active versus inactive SLE, they could demonstrate a stepwise progression of transcriptome alterations from inactive non-renal SLE to active non-renal and active renal SLE.

Parallel to molecular mapping, different groups have attempted dissection of the cellular constellation of the lupus kidney. In a recent conference abstract, Crickx et $a b^{67}$ showed that B cells with plasmablast attributes infiltrate the kidney of patients with active LN before therapeutic intervention, whereas the kidney of patients who failed immunosuppressive therapy with mycophenolate mofetil was enriched with long-lived plasma cells, introducing a prospect of cellular biomarkers of response to therapy. Advancing cellular approaches, Der et $a l^{68}$ for the Accelerating Medicines Partnership Rheumatoid Arthritis and SLE consortium recently published data from single-cell RNA sequencing on kidney biopsies from LN patients, demonstrating that high interferon response signature and fibrotic signature in tubular cells were associated with failure to respond to therapy and providing novel insights into histological differences across LN subtypes. Such techniques allow detailed characterisation of the cell populations in the kidney tissue, both infiltrating and resident immune cells as well as 
parenchymal cells, potentialising a deeper understanding of the pathophysiology underlying kidney inflammation in SLE in the forthcoming years and making the noninvasive biomarker mining in more accessible biological material, such as serum and urine, more relevant and more individualised. ${ }^{69} 70$

\section{PERSPECTIVES}

To summarise, the management of $\mathrm{LN}$ has improved during the last decades, but the imperative need for consensual outcome measures remains. In order to design trials with success potentiality, it is important to define clinically important short-term and long-term targets of therapeutic and non-therapeutic intervention. Ideally, an international task force comprising rheumatologists, nephrologists and patient advocates will soon be formed to serve this aim. The information gleaned from kidney biopsies at active renal disease and after completion of treatment may provide important insights in this direction. Moreover, novel insights of SLE and LN pathogenesis as well as insights obtained from genetic studies, transcriptomics and proteomics will have to be incorporated. $^{7172}$

Hopefully, we will soon identify clinical, molecular or genetic markers, or combinations thereof, which reliably reflect renal histopathology and portend the long-term renal outcome. Novel non-invasive imaging methods and employment of the evolving artificial intelligence in pattern recognition may also be helpful towards these goals. Undoubtedly, the molecular and cellular characterisation of SLE and LN will result in novel therapeutic modalities, maybe new taxonomy perspectives, and ultimately personalised management of the patients.

Contributors Manuscript draft: all authors. All authors read and critically revised the manuscript for intellectual content, approved its final version prior to submission and agree to be accountable for all aspects of the work.

Funding This work was supported by grants from the Swedish Rheumatism Association (R-859621), Professor Nanna Svartz Foundation (2019-00290), Ulla and Roland Gustafsson Foundation (2019-12), Region Stockholm, Karolinska Institutet (Sweden) and Fondation Saint-Luc and Fonds National de la Recherche Scientifique (Belgium).

Competing interests None declared.

Patient and public involvement Patients and/or the public were not involved in the design, or conduct, or reporting, or dissemination plans of this research. Patient consent for publication Not required.

Provenance and peer review Commissioned; internally peer reviewed.

Open access This is an open access article distributed in accordance with the Creative Commons Attribution Non Commercial (CC BY-NC 4.0) license, which permits others to distribute, remix, adapt, build upon this work non-commercially, and license their derivative works on different terms, provided the original work is properly cited, appropriate credit is given, any changes made indicated, and the use is non-commercial. See: http://creativecommons.org/licenses/by-nc/4.0/.

ORCID iD

Ioannis Parodis http://orcid.org/0000-0002-4875-5395

\section{REFERENCES}

1 Anders H-J, Saxena R, Zhao M-H, et al. Lupus nephritis. Nat Rev Dis Primers 2020;6:7.
2 Singh S, Saxena R. Lupus nephritis. Am J Med Sci 2009;337:451-60.

3 Pons-Estel GJ, Serrano R, Plasín MA, et al. Epidemiology and management of refractory lupus nephritis. Autoimmun Rev 2011:10:655-63.

4 Cervera R, Khamashta MA, Font J, et al. Morbidity and mortality in systemic lupus erythematosus during a 10-year period: a comparison of early and late manifestations in a cohort of 1,000 patients. Medicine 2003;82:299-308.

5 Appel GB, Contreras G, Dooley MA, et al. Mycophenolate mofetil versus cyclophosphamide for induction treatment of lupus nephritis. J Am Soc Nephrol 2009;20:1103-12.

6 Ginzler EM, Dooley MA, Aranow C, et al. Mycophenolate mofetil or intravenous cyclophosphamide for lupus nephritis. N Engl J Med 2005;353:2219-28.

7 Houssiau FA, Vasconcelos C, D'Cruz D, et al. Early response to immunosuppressive therapy predicts good renal outcome in lupus nephritis: lessons from long-term followup of patients in the EuroLupus nephritis trial. Arthritis Rheum 2004;50:3934-40.

8 Tektonidou MG, Dasgupta A, Ward MM. Risk of end-stage renal disease in patients with lupus nephritis, 1971-2015: a systematic review and Bayesian meta-analysis. Arthritis Rheumatol 2016;68:1432-41.

9 Houssiau FA, Vasconcelos C, D'Cruz D, et al. The 10-year follow-up data of the Euro-Lupus nephritis trial comparing low-dose and highdose intravenous cyclophosphamide. Ann Rheum Dis 2010;69:61-4.

10 Vandepapelière J, Aydin S, Cosyns J-P, et al. Prognosis of proliferative lupus nephritis subsets in the Louvain lupus nephritis inception cohort. Lupus 2014;23:159-65.

11 Zhang L, Lee G, Liu X, et al. Long-Term outcomes of end-stage kidney disease for patients with lupus nephritis. Kidney Int 2016:89:1337-45.

12 Houssiau FA, D'Cruz D, Sangle S, et al. Azathioprine versus mycophenolate mofetil for long-term immunosuppression in lupus nephritis: results from the maintain nephritis trial. Ann Rheum Dis 2010;69:2083-9.

13 Houssiau FA, Vasconcelos C, D'Cruz D, et al. Immunosuppressive therapy in lupus nephritis: the Euro-Lupus nephritis trial, a randomized trial of low-dose versus high-dose intravenous cyclophosphamide. Arthritis Rheum 2002;46:2121-31.

14 Tamirou F, Lauwerys BR, Dall'Era M, et al. A proteinuria cut-off level of $0.7 \mathrm{~g} /$ day after 12 months of treatment best predicts long-term renal outcome in lupus nephritis: data from the MAINTAIN Nephritis Trial. Lupus Sci Med 2015;2:e000123.

15 Dall'Era M, Cisternas MG, Smilek DE, et al. Predictors of long-term renal outcome in lupus nephritis trials: lessons learned from the Euro-Lupus nephritis cohort. Arthritis Rheumatol 2015;67:1305-13.

16 Korbet SM, Lewis EJ, Collaborative Study Group. Severe lupus nephritis: the predictive value of $a \geq 50 \%$ reduction in proteinuria at 6 months. Nephrol Dial Transplant 2013;28:2313-8.

17 Ugolini-Lopes MR, Seguro LPC, Castro MXF, et al. Early proteinuria response: a valid real-life situation predictor of long-term lupus renal outcome in an ethnically diverse group with severe biopsy-proven nephritis? Lupus Sci Med 2017;4:e000213.

18 Petri M, Orbai A-M, Alarcón GS, et al. Derivation and validation of the systemic lupus international collaborating clinics classification criteria for systemic lupus erythematosus. Arthritis Rheum 2012;64:2677-86.

19 Tan EM, Cohen AS, Fries JF, et al. The 1982 revised criteria for the classification of systemic lupus erythematosus. Arthritis Rheum 1982;25:1271-7.

20 Fogazzi GB, Saglimbeni L, Banfi G, et al. Urinary sediment features in proliferative and non-proliferative glomerular diseases. J Nephrol 2005;18:703-10.

21 Yuste C, Gutierrez E, Sevillano AM, et al. Pathogenesis of glomerular haematuria. World J Nephrol 2015;4:185-95.

22 Aringer M, Costenbader K, Daikh D, et al. European League against Rheumatism/American College of rheumatology classification criteria for systemic lupus erythematosus. Ann Rheum Dis 2019;2019:1151-9.

23 Hill GS, Delahousse M, Nochy D, et al. A new morphologic index for the evaluation of renal biopsies in lupus nephritis. Kidney Int 2000;58:1160-73.

24 Mackay M, Dall'Era M, Fishbein J, et al. Establishing surrogate kidney end points for lupus nephritis clinical trials: development and validation of a novel approach to predict future kidney outcomes. Arthritis Rheumatol 2019;71:411-9.

25 Bertsias GK, Tektonidou M, Amoura Z, et al. Joint European League against rheumatism and European renal AssociationEuropean dialysis and transplant association (EULAR/ERA-EDTA) recommendations for the management of adult and paediatric lupus nephritis. Ann Rheum Dis 2012;71:1771-82. 
26 Hahn BH, McMahon MA, Wilkinson A, et al. American College of rheumatology guidelines for screening, treatment, and management of lupus nephritis. Arthritis Care Res 2012;64:797-808

27 Bihl GR, Petri M, Fine DM. Kidney biopsy in lupus nephritis: look before you leap. Nephrol Dial Transplant 2006;21:1749-52.

28 Ward F, Bargman JM. Membranous lupus nephritis: the same, but different. Am J Kidney Dis 2016;68:954-66.

29 Bajema IM, Wilhelmus S, Alpers CE, et al. Revision of the International Society of Nephrology/Renal pathology Society classification for lupus nephritis: clarification of definitions, and modified National Institutes of health activity and chronicity indices. Kidney Int 2018;93:789-96.

30 Austin HA, Muenz LR, Joyce KM, et al. Prognostic factors in lupus nephritis. contribution of renal histologic data. Am J Med 1983;75:382-91.

31 Schwartz MM, Bernstein J, Hill GS, et al. Predictive value of renal pathology in diffuse proliferative lupus glomerulonephritis. lupus nephritis collaborative Study Group. Kidney Int 1989;36:891-6.

32 Daniel L, Sichez H, Giorgi R, et al. Tubular lesions and tubular cell adhesion molecules for the prognosis of lupus nephritis. Kidney Int 2001;60:2215-21.

33 Ferraccioli G, Romano G. Renal interstitial cells, proteinuria and progression of lupus nephritis: new frontiers for old factors. Lupus 2008;17:533-40.

34 Yu F, Wu L-H, Tan Y, et al. Tubulointerstitial lesions of patients with lupus nephritis classified by the 2003 International Society of nephrology and renal pathology Society system. Kidney Int 2010;77:820-9.

35 Hsieh C, Chang A, Brandt D, et al. Predicting outcomes of lupus nephritis with tubulointerstitial inflammation and scarring. Arthritis Care Res 2011;63:865-74.

36 Broder A, Mowrey WB, Khan HN, et al. Tubulointerstitial damage predicts end stage renal disease in lupus nephritis with preserved to moderately impaired renal function: a retrospective cohort study. Semin Arthritis Rheum 2018;47:545-51.

37 Clark MR, Trotter K, Chang A. The pathogenesis and therapeutic implications of tubulointerstitial inflammation in human lupus nephritis. Semin Nephrol 2015;35:455-64.

38 Eddy AA. Proteinuria and interstitial injury. Nephrol Dial Transplant 2004;19:277-81.

39 Ronda N, Cravedi P, Benozzi L, et al. Early proinflammatory activation of renal tubular cells by normal and pathologic IgG. Nephron Exp Nephrol 2005;100:e77-84.

40 Zheng L, Sinniah R, Hsu SI-H. Pathogenic role of NF-kappaB activation in tubulointerstitial inflammatory lesions in human lupus nephritis. J Histochem Cytochem 2008;56:517-29.

41 Theilig F. Spread of glomerular to tubulointerstitial disease with a focus on proteinuria. Ann Anat 2010;192:125-32.

42 Bonanni A, Vaglio A, Bruschi M, et al. Multi-antibody composition in lupus nephritis: isotype and antigen specificity make the difference. Autoimmun Rev 2015;14:692-702.

43 Yap DYH, Yung S, Zhang Q, et al. Serum level of proximal renal tubular epithelial cell-binding immunoglobulin $\mathrm{G}$ in patients with lupus nephritis. Lupus 2016;25:46-53.

44 Anders H-J. Re-biopsy in lupus nephritis. Ann Trans/ Med 2018;6:S41.

45 Zickert A, Sundelin B, Svenungsson E, et al. Role of early repeated renal biopsies in lupus nephritis. Lupus Sci Med 2014;1:e000018.

46 De Rosa M, Azzato F, Toblli JE, et al. A prospective observational cohort study highlights kidney biopsy findings of lupus nephritis patients in remission who flare following withdrawal of maintenance therapy. Kidney Int 2018;94:788-94.

47 Malvar A, Pirruccio P, Alberton V, et al. Histologic versus clinical remission in proliferative lupus nephritis. Nephrol Dial Transplant 2017;32:1338-44.

48 Piñeiro GJ, Arrizabalaga P, Solé M, et al. Repeated Renal Biopsy - A Predictive Tool to Assess the Probability of Renal Flare in Lupus Nephritis. Am J Nephrol 2016;44:439-46.

49 Arends S, Grootscholten C, Derksen RHWM, et al. Long-Term follow-up of a randomised controlled trial of azathioprine/ methylprednisolone versus cyclophosphamide in patients with proliferative lupus nephritis. Ann Rheum Dis 2012;71:966-73.
50 Hill GS, Delahousse M, Nochy D, et al. Predictive power of the second renal biopsy in lupus nephritis: significance of macrophages. Kidney Int 2001;59:304-16.

51 Mok CC. Towards new avenues in the management of lupus glomerulonephritis. Nat Rev Rheumatol 2016;12:221-34.

52 Treamtrakanpon W, Tantivitayakul P, Benjachat T, et al. April, a proliferation-inducing ligand, as a potential marker of lupus nephritis. Arthritis Res Ther 2012;14:R252.

53 Parodis I, Zickert A, Sundelin B, et al. Evaluation of B lymphocyte stimulator and a proliferation inducing ligand as candidate biomarkers in lupus nephritis based on clinical and histopathological outcome following induction therapy. Lupus Sci Med 2015;2:e000061.

54 Zickert A, Oke V, Parodis I, et al. Interferon (IFN)- $\lambda$ is a potentia mediator in lupus nephritis. Lupus Sci Med 2016;3:e000170.

55 Parodis I, Ding H, Zickert A, et al. Serum soluble tumour necrosis factor receptor-2 (sTNFR2) as a biomarker of kidney tissue damage and long-term renal outcome in lupus nephritis. Scand J Rheumatol 2017;46:263-72.

56 Parodis I, Ding H, Zickert A, et al. Serum Axl predicts histologybased response to induction therapy and long-term renal outcome in lupus nephritis. PLoS One 2019;14:e0212068.

57 Mok CC, Ding HH, Kharboutli M, et al. Axl, ferritin, insulin-like growth factor binding protein 2, and tumor necrosis factor receptor type II as biomarkers in systemic lupus erythematosus. Arthritis Care Res 2016:68:1303-9.

$58 \mathrm{Wu}$ T, Ding H, Han J, et al. Antibody-Array-Based proteomic screening of serum markers in systemic lupus erythematosus: a discovery study. J Proteome Res 2016;15:2102-14.

59 Reyes-Thomas J, Blanco I, Putterman C. Urinary biomarkers in lupus nephritis. Clin Rev Allergy Immunol 2011:40:138-50.

60 Mok CC, Soliman S, Ho LY, et al. Urinary angiostatin, CXCL4 and VCAM-1 as biomarkers of lupus nephritis. Arthritis Res Ther 2018;20:6.

61 Wu T, Xie C, Wang HW, et al. Elevated urinary VCAM-1, P-selectin, soluble TNF receptor-1, and CXC chemokine ligand 16 in multiple murine lupus strains and human lupus nephritis. $J$ Immunol 2007;179:7166-75.

62 Parodis I, Gokaraju S, Zickert A, et al. Alcam and VCAM-1 as urine biomarkers of activity and long-term renal outcome in systemic lupus erythematosus. Rheumatology 2019. doi:10.1093/rheumatology/ kez528. [Epub ahead of print: 13 Nov 2019].

64 Arazi A, Rao DA, Berthier CC, et al. The immune cell landscape in kidneys of patients with lupus nephritis. Nat Immunol 2019;20:902-14.

64 Pamfil C, Makowska Z, De Groof A, et al. Intrarenal activation of adaptive immune effectors is associated with tubular damage and impaired renal function in lupus nephritis. Ann Rheum Dis 2018;77:1782-9.

65 Parikh SV, Malvar A, Song H, et al. Molecular imaging of the kidney in lupus nephritis to characterize response to treatment. Trans/ Res 2017;182:1-13.

66 Panousis NI, Bertsias GK, Ongen $\mathrm{H}$, et al. Combined genetic and transcriptome analysis of patients with SLE: distinct, targetable signatures for susceptibility and severity. Ann Rheum Dis 2019;78:1079-89.

67 Crickx E, Tamirou F, Huscenot T, et al. THU0211 evolution of kidney antibody secreting cells molecular sugnature in lupus patients with active nephritis upon immunosuppressive therapy. Ann Rheum Dis 2019;78:384

68 Der $\mathrm{E}$, Suryawanshi $\mathrm{H}$, Morozov $\mathrm{P}$, et al. Tubular cell and keratinocyte single-cell transcriptomics applied to lupus nephritis reveal type I IFN and fibrosis relevant pathways. Nat Immunol 2019;20:915-27.

69 Der E, Suryawanshi H, Buyon J, et al. Single-Cell RNA sequencing for the study of lupus nephritis. Lupus Sci Med 2019;6:e000329.

70 Rao DA, Arazi A, Wofsy D, et al. Design and application of single-cell RNA sequencing to study kidney immune cells in lupus nephritis. Nat Rev Nephrol 2019. doi:10.1038/s41581-019-0232-6. [Epub ahead of print: 18 Dec 2019].

71 Yu F, Haas M, Glassock R, et al. Redefining lupus nephritis: clinical implications of pathophysiologic subtypes. Nat Rev Nephrol 2017:13:483-95.

72 Alarcón-Riquelme ME. New attempts to define and clarify lupus. Curr Rheumatol Rep 2019;21:11. 